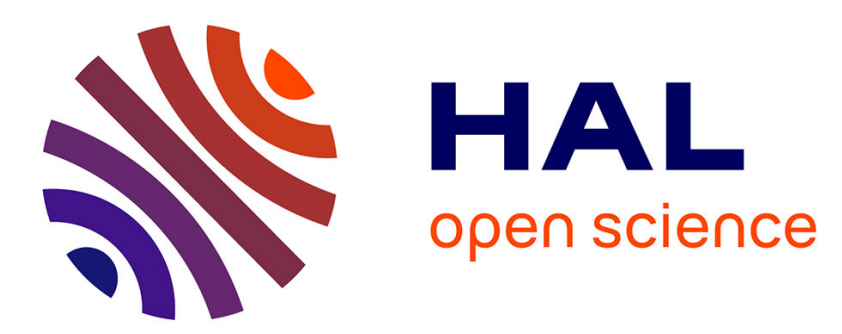

\title{
General Optimality Conditions for Constrained Convex Control Problems
}

Maïtine Bergounioux, Dan Tiba

\section{To cite this version:}

Maïtine Bergounioux, Dan Tiba. General Optimality Conditions for Constrained Convex Control Problems. SIAM Journal on Control and Optimization, 1996, 34, $\mathrm{n}^{\circ} 2$, pp. 698-711. hal-00023059

\section{HAL Id: hal-00023059 \\ https://hal.science/hal-00023059}

Submitted on 19 Apr 2006

HAL is a multi-disciplinary open access archive for the deposit and dissemination of scientific research documents, whether they are published or not. The documents may come from teaching and research institutions in France or abroad, or from public or private research centers.
L'archive ouverte pluridisciplinaire $\mathbf{H A L}$, est destinée au dépôt et à la diffusion de documents scientifiques de niveau recherche, publiés ou non, émanant des établissements d'enseignement et de recherche français ou étrangers, des laboratoires publics ou privés. 


\title{
General Optimality Conditions for Constrained Convex Control Problems
}

\author{
Maïtine Bergounioux \\ Département de Mathématiques \\ URA CNRS 1803 - Université d'Orléans \\ B.P. 6759 - 45067 Orléans Cedex 2
}

France

\author{
Dan Tiba \\ Institute for Mathematics \\ Romanian Academy of Sciences \\ 70700 Bucuresti P.O.Box 1-764 \\ Romania
}

\begin{abstract}
In this paper we investigate some optimal convex control problems, with mixed constraints on the state and the control. We give a general condition which allows to set optimality conditions for non qualified problems (in the Slater sense). Then we give some applications and examples involving generalized bang-bang results.
\end{abstract}

Keywords: Optimal Control with Mixed Constraints, Qualification Condition, Optimality Conditions

AMS subject classification. 49B22

\section{Formulation of the Problem}

Let $V \subset H \subset V^{\prime}$ compactly and densely be Hilbert spaces, $A(t): V \rightarrow V^{\prime}, B: U \rightarrow V^{\prime}$ be linear bounded operators ( $\mathrm{U}$ is another non trivial Hilbert space) and $L: L^{2}(0, T ; H \times U) \rightarrow$ $\mathbb{R}, l: H \rightarrow \mathbb{R}$ be convex, continuous mappings.

We consider the following optimal control problem :

(P) $\quad \operatorname{Min}\{L(x, u)+l(x(T))\}$

subject to

$$
\begin{gathered}
\left.x^{\prime}(t)+A(t) x(t)=B u(t)+f(t) \quad \text { a.e. in }\right] 0, T[ \\
{[x, u] \in D \subset \mathcal{X} \times L^{2}(0, T ; U), \text { closed convex subset }}
\end{gathered}
$$

where

- $\mathcal{X}=L^{2}(0, T ; V) \cap W^{1,2}\left(0, T ; V^{\prime}\right)$,

- $f \in L^{2}\left(0, T ; V^{\prime}\right)$,

- $L$ is coercive in the sense

$$
\begin{aligned}
& \exists c(\text { a generic constant })>0 \text { such that }, \\
& \forall[y, u] \in L^{2}(0, T ; H \times U) \quad L(y, u)>c\|u\|_{L^{2}(0, T ; U)}^{2}-c,
\end{aligned}
$$


- $\forall z \in V \quad t \mapsto A(t) z$ is $V^{\prime}$-measurable on $] 0, T[$, and

$$
\begin{gathered}
\forall z \in V \quad\|A(t) z\|_{V^{\prime}} \leq c\|z\|_{V}, \\
\exists \alpha, \exists \beta>0, \quad \forall z \in V \quad\langle A(t) z, z\rangle_{V \times V^{\prime}}+\alpha\|z\|_{H}^{2} \geq \beta\|z\|_{V}^{2} .
\end{gathered}
$$

We assume the initial condition $x(0)=x^{o} \in H$ and the possible final restrictions are included in the definition of $D$. The evolution equation (1.1) has a unique solution $x \in \mathcal{X}$ for any $u \in L^{2}(0, T ; U)$, by theorem 4.5, Barbu and Precupanu [3], Ch. 1 .

Moreover, by condition (1.3), it is a standard argument to show that $(P)$ has at least one optimal pair (denoted $\left[x^{*}, u^{*}\right]$ ) in $D$ if some admissibility assumption is fullfilled :

$$
\exists[x, u] \in D \text { such that } T(x, u)=0 \text {, }
$$

with

$$
\forall[x, u] \in \mathcal{X} \times L^{2}(0, T ; U) \quad T(x, u)=x^{\prime}+A(t) x-B u-f .
$$

The problem $(P)$ is a generalization of the Bolza optimal control problem studied by Barbu and Precupanu [3], Ch.4, both with respect to the cost functional and with respect to the form of the mixed constraints. The continuity hypothesis on $L$ and $l$ is quite restrictive, but as we keep the constraints separately (i.e. we do not include them into the cost via the indicator function of $D$ ), then the class of examples is very large.

For state constrained control problems, one usually assume a Slater type interiority condition. In the general setting of (1.2), it takes the form :

$$
\exists[\bar{x}, \bar{u}] \text { feasible for }(P) \text { such that } \bar{x} \in \operatorname{int}\{y \in \mathcal{X} \mid[y, \bar{u}] \in D\} \text { in } \mathcal{C}(0, T ; H),
$$

and it has very severe implications on the set of possible applications.

It is our main concern to weaken this classical qualification constraint. Namely, instead of $(\mathcal{S})$, we shall suppose that

$$
\begin{gathered}
\exists \mathcal{M} \subset D \text { bounded in } \mathcal{C}(0, T ; H) \times L^{2}(0, T ; U) \\
\text { such that } 0 \in \operatorname{int} T(\mathcal{M}) \text { in } L^{2}\left(0, T ; V^{\prime}\right) .
\end{gathered}
$$

Let us first notice that 0 appears naturally in $(\mathcal{H})$ since the problem constraint is expressed as $T(x, u)=0$. Moreover, the elements (pairs) of the set $\mathcal{M}$ need not be feasible for $(P)$.

We first compare the two conditions $(\mathcal{S})$ and $(\mathcal{H})$; the following proposition proves that $(\mathcal{S})$ is always stronger than $(\mathcal{H})$.

Proposition $1.1(\mathcal{S}) \Rightarrow(\mathcal{H})$.

Proof.- Thanks to $(\mathcal{S}),(1.1)$ and $(1.2)$ we have

$$
\left.\bar{x}^{\prime}+A(t) \bar{x}=B \bar{u}+f, \bar{x}(0)=x^{o}, \text { a.e. in }\right] 0, T[.
$$

Let $\rho>0$ and $\xi \in L^{2}\left(0, T ; V^{\prime}\right)$ such that $\|\xi\|_{L^{2}\left(0, T ; V^{\prime}\right)}=1$. We denote by $x_{\xi}$ the solution of

$$
\left.x_{\xi}^{\prime}+A(t) x_{\xi}=B \bar{u}+f+\rho \xi, x_{\xi}(0)=x^{o}, \text { a.e. in }\right] 0, T[\text {. }
$$


Taking the difference between (1.7) and (1.8), we get that

$$
\left\|\bar{x}-x_{\xi}\right\|_{\mathcal{X}} \leq k \rho,
$$

with $k$ independent of $\xi$. Then, if $\rho$ is small enough, $(\mathcal{S})$ gives $\left[x_{\xi}, \bar{u}\right] \in D$, for all $\xi \in$ $L^{2}\left(0, T ; V^{\prime}\right)$ such that $\|\xi\|_{L^{2}\left(0, T ; V^{\prime}\right)}=1$. Here we set

$$
\mathcal{M}=\operatorname{conv}\left(\left\{\left[x_{\xi}, \bar{u}\right] \mid \xi \in L^{2}\left(0, T ; V^{\prime}\right),\|\xi\|_{L^{2}\left(0, T ; V^{\prime}\right)}=1\right\}\right),
$$

where $\operatorname{conv}(\mathrm{E})$ is the convex hull of the set $\mathrm{E}$, and the proof is finished.

Remark 1.1 Assume that $U \subset V^{\prime}$ continuously and $B: U \rightarrow V^{\prime}$ is the canonical injection. Then one ask an interiority assumption with respect to the control of the type :

$$
\begin{gathered}
\exists[\tilde{x}, \tilde{u}] \text { feasible for }(P) \text { such that } \\
\text { Int }\{u \in U \mid[\tilde{x}, u] \in D\} \text { is non empty in } L^{2}\left(0, T ; V^{\prime}\right) .
\end{gathered}
$$

This again implies $(\mathcal{H})$ by an argument as above. In this case the Slater condition need not be fulfilled, that is to say that the condition $(\mathcal{H})$ is strictly weaker than $(\mathcal{S})$.

Condition $(\mathcal{H})$ or its weaker variant $\left(\mathcal{H}^{\prime}\right)$ from section 3 . may be mainly compared with the Zowe and Kurcyusz [18] condition in the mathematical programming theory. This was previously used in abstract control problems by Tröltzsch [16, 17], combined with interiority type assumptions at the level of applications. In the examples of section 3., we show that the interior of the constraint set may be empty even in the uniform topology, but the argument still applies.

In the recent work of Barbu and Pavel [2] another case of empty interior constraints is discussed for optimal control problems governed by periodic evolution equations and by a different method. Our approach is based on the penalization of the only state system rather than of both the state system and the constraints (as in Bonnans and Casas [8]); the constraints are kept explicitly throughout the proof. This is quite a classical philosophy in connection to Lagrange multipliers techniques (see for instance the monograph of Tikhomirov [15]). In the setting of partial differential equations, it has been extensively exploited in the books of Lions [12] or Tiba [14], Ch.2, in connection to nonlinear singular control problems. Recently Bergounioux $[5,6]$ has applied this method to control problems with state constraints governed by elliptic systems and Bergounioux, Männikkö and Tiba [7] have studied some examples of parabolic control problems. Applications of the obtained optimality system to augmented Lagrangian algorithms were also indicated.

Finally, we point out that the technique used in the next section puts into evidence with full accuracy the relationship between the operator $T$ and the set $D$ of constraints.

\section{The Optimality System}

We define the penalized problem as following

$\left(P_{\varepsilon}\right) \quad \operatorname{Min}\left\{L_{\varepsilon}(x, u)+l_{\varepsilon}(x(T))+\frac{1}{2} \int_{0}^{T}\left\|u-u^{*}\right\|_{U}^{2} d t+\frac{1}{2 \varepsilon} \int_{0}^{T}\left\|x^{\prime}+A x-B u-f\right\|_{V^{\prime}}^{2} d t\right\}$

over all $[x, u] \in D$. It should be noted that the first integral is an "adapted" penalization term according to Barbu [1], while $L_{\varepsilon}$ and $l_{\varepsilon}$ are the Moreau-Yosida regularization of the convex mappings $L$ and $l$. 
Remark 2.1 Let us briefly recall what the Moreau-Yosida regularization is. Let $f$ be a proper, convex mapping on a Banach space $X$. For anay $\varepsilon>0$, the Moreau-Yosida regularization of $f$ is :

$$
\forall x \in X \quad f_{\varepsilon}(x)=\inf \left\{\frac{1}{2 \varepsilon}\|x-y\|_{X}^{2}+f(y) \mid y \in X\right\} .
$$

A thorough study of the properties of $L_{\varepsilon}$ and $l_{\varepsilon}$ may be found in Barbu and Precupanu [3], ch. 2. We shall recall some of them when needed in the text.

The existence of a unique optimal pair $\left[x_{\varepsilon}, u_{\varepsilon}\right]$ is obvious. We also denote

$$
r_{\varepsilon}=\frac{1}{\varepsilon} J^{-1}\left(x_{\varepsilon}^{\prime}+A x_{\varepsilon}-B u_{\varepsilon}-f\right) \in L^{2}(0, T ; V)
$$

where $J: V \rightarrow V^{\prime}$ is the canonical isomorphism.

Proposition 2.1 We have

$$
\begin{gathered}
x_{\varepsilon} \rightarrow x^{*} \quad \text { strongly in } \mathcal{X}, \\
u_{\varepsilon} \rightarrow u^{*} \quad \text { strongly in } L^{2}(0, T ; U), \\
\varepsilon^{\frac{1}{2}} r_{\varepsilon} \quad \text { is bounded in } L^{2}(0, T ; V),
\end{gathered}
$$

Proof.- The optimality of the pair $\left[x^{*}, u^{*}\right]$ and the properties of the convex regularized mappings give

$$
\begin{gathered}
L_{\varepsilon}\left(x_{\varepsilon}, u_{\varepsilon}\right)+l_{\varepsilon}\left(x_{\varepsilon}(T)\right)+\frac{1}{2} \int_{0}^{T}\left\|u_{\varepsilon}-u^{*}\right\|_{U}^{2} d t+\frac{1}{2 \varepsilon} \int_{0}^{T}\left\|x_{\varepsilon}^{\prime}+A(t) x_{\varepsilon}-B u_{\varepsilon}-f\right\|_{V^{\prime}}^{2} d t \\
\leq L_{\varepsilon}\left(x^{*}, u^{*}\right)+l_{\varepsilon}\left(x^{*}(T)\right) \leq L\left(x^{*}, u^{*}\right)+l\left(x^{*}(T)\right) .
\end{gathered}
$$

With the coercivity assumption (1.3), the relation (2.4) gives

$$
l_{\varepsilon}\left(x_{\varepsilon}(T)\right)+\frac{1}{2} \int_{0}^{T}\left\|u_{\varepsilon}-u^{*}\right\|_{U}^{2} d t+\frac{1}{2 \varepsilon} \int_{0}^{T}\left\|x_{\varepsilon}^{\prime}+A(t) x_{\varepsilon}-B u_{\varepsilon}-f\right\|_{V^{\prime}}^{2} d t \leq c .
$$

Moreover $l_{\varepsilon}$ is lower bounded by an affine mapping uniformly with respect to $\varepsilon>0$ so that

$$
\frac{1}{2} \int_{0}^{T}\left\|u_{\varepsilon}-u^{*}\right\|_{U}^{2} d t+\frac{1}{2 \varepsilon} \int_{0}^{T}\left\|x_{\varepsilon}^{\prime}+A(t) x_{\varepsilon}-B u_{\varepsilon}-f\right\|_{V^{\prime}}^{2} d t \leq c+c\left\|x_{\varepsilon}(T)\right\|_{H} .
$$

As the initial condition is contained in $D$ and the dependence from the right hand side as defined by (1.1) is sublinear, then we see that $\left(u_{\varepsilon}\right)$ is bounded in $L^{2}(0, T ; U), x_{\varepsilon}$ is bounded in $\mathcal{X}$ and $\varepsilon^{\frac{1}{2}} r_{\varepsilon}$ is bounded in $L^{2}(0, T ; V)$.

We note $[\hat{x}, \hat{u}]$ their weak limit on a subsequence. Since

$$
x_{\varepsilon}^{\prime}+A(t) x_{\varepsilon}=B u_{\varepsilon}+f+\varepsilon J\left(r_{\varepsilon}\right),
$$

we can pass to the limit and $[\hat{x}, \hat{u}]$ is an feasible pair for $(P)$. We have

$$
L_{\varepsilon}\left(x_{\varepsilon}, u_{\varepsilon}\right)=L\left((I+\varepsilon \partial L)^{-1}\left(x_{\varepsilon}, u_{\varepsilon}\right)\right)+\frac{1}{2 \varepsilon}\left\|\left[x_{\varepsilon}, u_{\varepsilon}\right]-(I+\varepsilon \partial L)^{-1}\left(x_{\varepsilon}, u_{\varepsilon}\right)\right\|_{H \times U}^{2},
$$


where $\mathrm{I}$ is the identity in $H \times U$. Coming back to $(2.4)$, we get easily that $L_{\varepsilon}\left(x_{\varepsilon}, u_{\varepsilon}\right)$ is bounded so $(I+\varepsilon \partial L)^{-1}\left(x_{\varepsilon}, u_{\varepsilon}\right) \rightarrow[\hat{x}, \hat{u}]$ weakly in $L^{2}(0, T ; H \times U)$ by the above formula. Taking in account the weak lower semicontinuity of $L$ and $l$ we can pass to the limit in $(2.4)$ :

$$
L(\hat{x}, \hat{u})+l(\hat{x}(T))+\frac{1}{2} \int_{0}^{T}\left\|\hat{u}-u^{*}\right\|_{U}^{2} d t \leq L\left(x^{*}, u^{*}\right)+l\left(x^{*}(T)\right) .
$$

Then $\hat{u}=u^{*}, \hat{x}=x^{*}$ and we have (2.2) and (2.1) by a strong convergence criterion in Hilbert spaces.

Proposition 2.2 We have the following first order optimality condition :

$$
\begin{aligned}
&\left\langle\nabla L_{\varepsilon}\left(x_{\varepsilon}, u_{\varepsilon}\right),\left[x_{\varepsilon}, u_{\varepsilon}\right]-[x, u]\right\rangle_{L^{2}(0, T ; H \times U)}+\left\langle\nabla l_{\varepsilon}\left(x_{\varepsilon}(T)\right), x_{\varepsilon}(T)-x(T)\right\rangle_{H} \\
& \quad+\int_{0}^{T}\left\langle u_{\varepsilon}-u, u_{\varepsilon}-u^{*}\right\rangle_{U} d t-\int_{0}^{T}\left\langle x^{\prime}+A x-B u-f, J\left(r_{\varepsilon}\right)\right\rangle_{V^{\prime}} d t \quad \leq 0
\end{aligned}
$$

for any $[x, u]$ in $D$. (Here $\nabla$ denotes the Gâteaux derivative.)

This is a standard result in the optimization of convex differentiable functionals (see Lions for instance [11], Ch.1) and $J\left(r_{\varepsilon}\right)$ plays the role of a Lagrange multiplier.

Proof.- We make feasible variations in $x$ and $u$ :

$$
\begin{aligned}
& L_{\varepsilon}\left(x_{\varepsilon}, u_{\varepsilon}\right)+l_{\varepsilon}\left(x_{\varepsilon}(T)\right)+\frac{1}{2} \int_{0}^{T}\left\|u_{\varepsilon}-u^{*}\right\|_{U}^{2} d t+\frac{1}{2 \varepsilon} \int_{0}^{T}\left\|x_{\varepsilon}^{\prime}+A x_{\varepsilon}-B u_{\varepsilon}-f\right\|_{V^{\prime}}^{2} d t \\
\leq & L_{\varepsilon}\left(x_{s}, u_{s}\right)+l_{\varepsilon}\left(x_{s}(T)\right)+\frac{1}{2} \int_{0}^{T}\left\|u_{s}-u^{*}\right\|_{U}^{2} d t+\frac{1}{2 \varepsilon} \int_{0}^{T}\left\|x_{s}^{\prime}+A x_{s}-B u_{s}-f\right\|_{V^{\prime}}^{2} d t,
\end{aligned}
$$

where $\left.\left.x_{s}=x_{\varepsilon}+s\left(x-x_{\varepsilon}\right), u_{s}=u_{\varepsilon}+s\left(u-u_{\varepsilon}\right), s \in\right] 0,1\right]$ and $[x, u] \in D$ arbitrary.

Passing all the terms to the left hand side, dividing by $s>0$ and letting $s$ tend to 0 , we obtain

$$
\begin{aligned}
& \left\langle\nabla L_{\varepsilon}\left(x_{\varepsilon}, u_{\varepsilon}\right),\left[x_{\varepsilon}, u_{\varepsilon}\right]-[x, u]\right\rangle_{L^{2}(0, T ; H \times U)}+\left\langle\nabla l_{\varepsilon}\left(x_{\varepsilon}(T)\right), x_{\varepsilon}(T)-x(T)\right\rangle_{H} \\
+ & \int_{0}^{T}\left\langle u_{\varepsilon}-u, u_{\varepsilon}-u^{*}\right\rangle_{U} d t-\int_{0}^{T}\left\langle x^{\prime}+A x-B u-f-\varepsilon J\left(r_{\varepsilon}\right), J\left(r_{\varepsilon}\right)\right\rangle_{V^{\prime}} d t \leq 0
\end{aligned}
$$

for any $[x, u]$ in $D$. Then (2.5) follows from (2.6) since $\varepsilon\left\|J\left(r_{\varepsilon}\right)\right\|_{L^{2}\left(0, T ; V^{\prime}\right)}^{2} \geq 0$.

(We have also used the properties of $J\left(r_{\varepsilon}\right)$.)

Remark 2.2 The condition (2.6) is also sufficient for optimality in (P). We can reexpress (2.5) as

$$
\begin{aligned}
&\left\langle\nabla L_{\varepsilon}\left(x_{\varepsilon}, u_{\varepsilon}\right),\left[x_{\varepsilon}, u_{\varepsilon}\right]-[x, u]\right\rangle_{L^{2}(0, T ; H \times U)}+\left\langle\nabla l_{\varepsilon}\left(x_{\varepsilon}(T)\right), x_{\varepsilon}(T)-x(T)\right\rangle_{H} \\
& \quad+\int_{0}^{T}\left\langle u_{\varepsilon}-u, u_{\varepsilon}-u^{*}\right\rangle_{U} d t-\int_{0}^{T}\left\langle x^{\prime}+A x-B u-f, r_{\varepsilon}\right\rangle_{V^{\prime} \times V} d t \quad \leq 0
\end{aligned}
$$

for any $[x, u]$ in $D$. 
Now we define the simplified adjoint system

$$
\begin{gathered}
-p_{\varepsilon}^{\prime}+A^{*}(t) p_{\varepsilon}=\nabla_{x} L_{\varepsilon}\left(x_{\varepsilon}, u_{\varepsilon}\right), \\
p_{\varepsilon}(T)=\nabla l_{\varepsilon}\left(x_{\varepsilon}(T)\right),
\end{gathered}
$$

where $A^{*}$ denotes the adjoint operator of $A$.

Multiply (2.8) by $x_{\varepsilon}-x$, for any $x$ in $\mathcal{X}$ such that $x(0)=x^{o}$, and integrate by parts we get

$$
\begin{aligned}
\left\langle\nabla_{x} L_{\varepsilon}\left(x_{\varepsilon}, u_{\varepsilon}\right), x_{\varepsilon}-x\right\rangle_{L^{2}(0, T ; H)}= & \int_{0}^{T}\left\langle-p_{\varepsilon}^{\prime}+A^{*} p_{\varepsilon}, x_{\varepsilon}-x\right\rangle_{H} d t \\
= & \int_{0}^{T}\left\langle x_{\varepsilon}^{\prime}-x^{\prime}+A\left(x_{\varepsilon}-x\right), p_{\varepsilon}\right\rangle_{V^{\prime} \times V} d t \\
& -\left\langle\nabla l_{\varepsilon}\left(x_{\varepsilon}(T)\right), x_{\varepsilon}(T)-x(T)\right\rangle_{H} .
\end{aligned}
$$

Replacing (2.10) in (2.6), we obtain the equivalent form

$$
\begin{gathered}
\left\langle\nabla_{u} L_{\varepsilon}\left(x_{\varepsilon}, u_{\varepsilon}\right), u_{\varepsilon}-u\right\rangle_{L^{2}(0, T ; U)}+\int_{0}^{T}\left\langle x_{\varepsilon}^{\prime}-x^{\prime}+A\left(x_{\varepsilon}-x\right), p_{\varepsilon}\right\rangle_{V^{\prime} \times V} d t \\
+\int_{0}^{T}\left\langle u_{\varepsilon}-u, u_{\varepsilon}-u^{*}\right\rangle_{U} d t-\int_{0}^{T}\left\langle x^{\prime}+A x-B u-f, J\left(r_{\varepsilon}\right)\right\rangle_{V^{\prime}} d t \\
\leq-\varepsilon\left\|J\left(r_{\varepsilon}\right)\right\|_{L^{2}\left(0, T ; V^{\prime}\right)}^{2} \leq 0,
\end{gathered}
$$

for any $[x, u]$ in $D$.

Taking in turn $u=u_{\varepsilon}, x=x_{\varepsilon}$, a short computation provides the following decoupled system :

$$
\begin{gathered}
\int_{0}^{T}\left\langle x_{\varepsilon}^{\prime}-x^{\prime}+A x_{\varepsilon}-A x, p_{\varepsilon}+r_{\varepsilon}\right\rangle_{V^{\prime} \times V} d t \leq 0 \\
\int_{0}^{T}\left\langle u_{\varepsilon}-u, u_{\varepsilon}-u^{*}\right\rangle_{U} d t+\left\langle\nabla_{u} L_{\varepsilon}\left(x_{\varepsilon}, u_{\varepsilon}\right), u_{\varepsilon}-u\right\rangle_{L^{2}(0, T ; U)} \\
-\int_{0}^{T}\left\langle u_{\varepsilon}-u, B^{*} J\left(r_{\varepsilon}\right)\right\rangle_{U} d t \leq 0
\end{gathered}
$$

for any $[x, u]$ such that $\left[x, u_{\varepsilon}\right] \in D$ and $\left[x_{\varepsilon}, u\right] \in D$.

Remark 2.3 The relations (2.8)-(2.9) and (2.12)-(2.13) give the optimality conditions for the problem $\left(P_{\varepsilon}\right)$ in a more usual form. In particular, if $D=K \times U_{a d}$ (i.e. the constraints are separate) and if $\mathcal{N}\left(u_{\varepsilon}\right)$ denotes the normal cone to the control constraints set at $u_{\varepsilon}$, that is

$$
\mathcal{N}\left(u_{\varepsilon}\right)=\left\{z \in L^{2}(0, T ; U) \mid\left\langle z, u_{\varepsilon}-u\right\rangle_{L^{2}(0, T ; U)} \geq 0, \forall u \in U_{a d}\right\}
$$

then (2.13) becomes

$$
\nabla_{u} L_{\varepsilon}\left(x_{\varepsilon}, u_{\varepsilon}\right)+u_{\varepsilon}-u^{*}+\mathcal{N}\left(u_{\varepsilon}\right) \ni B^{*} J\left(r_{\varepsilon}\right)
$$

which is a standard form of the Pontryagin maximum principle (Barbu and Precupanu [3], Ch. IV, Tiba [14], Ch. II). 
Proposition 2.3 On a subsequence, we have :

$$
\begin{gathered}
\nabla l_{\varepsilon}\left(x_{\varepsilon}(T)\right) \rightarrow w \in \partial l\left(x^{*}(T)\right) \text { weakly in } H, \\
\nabla L_{\varepsilon}\left(x_{\varepsilon}, u_{\varepsilon}\right) \rightarrow\left(w_{1}, w_{2}\right) \in \partial L\left(x^{*}, u^{*}\right) \text { weakly in } L^{2}(0, T ; H \times U), \\
p_{\varepsilon} \rightarrow p^{*} \text { strongly in } \mathcal{C}(0, T ; H),
\end{gathered}
$$

where $p^{*}$ is the solution of the simplified adjoint system

$$
\begin{gathered}
-\frac{d p^{*}}{d t}+A^{*} p^{*}=w_{1}, \\
p^{*}(T)=w .
\end{gathered}
$$

Proof.- We have : $\nabla L_{\varepsilon}\left(x_{\varepsilon}, u_{\varepsilon}\right) \in \partial L\left((I+\varepsilon \partial L)^{-1}\right)\left(x_{\varepsilon}, u_{\varepsilon}\right)$. With a similar argument to the one of the proof of proposition 2.1, it yields that $\left.(I+\varepsilon \partial L)^{-1}\right)\left(x_{\varepsilon}, u_{\varepsilon}\right)$ strongly converges to $\left[x^{*}, u^{*}\right]$ in $L^{2}(0, T ; H \times U)$. As $L$ is continuous, it is everywhere sub-differentiable and $\partial L$ is locally bounded. Then $\nabla L_{\varepsilon}\left(x_{\varepsilon}, u_{\varepsilon}\right)$ is bounded in $L^{2}(0, T ; H \times U)$ and $(2.15)$ is a consequence of the demiclosedness of maximal monotone operators.

The argument is the same for relation (2.14) and (2.16)-(2.17)- (2.18) may be obtained taking the limit in (2.8)-(2.9).

Proposition 2.4 Under the hypothesis $(\mathcal{H}),\left(r_{\varepsilon}\right)$ is bounded in $L^{2}(0, T ; V)$ and $r_{\varepsilon} \rightarrow r^{*}$ on a subsequence, weakly in $L^{2}(0, T ; V)$.

Proof.- We use the relation (2.7); we take test functions $\left[x_{\xi}, u_{\xi}\right] \in \mathcal{M} \subset D$ such that

$$
T\left(x_{\xi}, u_{\xi}\right)=\rho \xi
$$

for any $\xi \in L^{2}\left(0, T ; V^{\prime}\right)$ such that $\|\xi\|_{L^{2}\left(0, T ; V^{\prime}\right)}=1$ and for some $\rho>0$. The boundedness of $\mathcal{M}$ and propositions (2.1) and (2.3) allow to infer

$$
\rho \int_{0}^{T}\left\langle\xi, r_{\varepsilon}\right\rangle_{V^{\prime} \times V} d t \leq c
$$

where $c$ is an absolute constant independent of $\varepsilon>0$ and $\xi$.

We have finally the following theorem :

Theorem 2.1 If the pair $\left[x^{*}, u^{*}\right]$ is optimal for the problem $(P)$, then

$$
\begin{gathered}
\int_{0}^{T}\left\langle x^{* \prime}-x^{\prime}+A x^{*}-A x, p^{*}+r^{*}\right\rangle_{V^{\prime} \times V} d t \leq 0, \\
\left\langle w_{2}, u^{*}-u\right\rangle_{L^{2}(0, T ; U)}-\int_{0}^{T}\left\langle u^{*}-u, B^{*} J^{-1}\left(r^{*}\right)\right\rangle_{U} d t \leq 0,
\end{gathered}
$$

for any $[x, u]$ such that $\left[x, u^{*}\right] \in D$ and $\left[x^{*}, u\right] \in D$.

Moreover the inequality summing (2.19) and (2.20) is valid for any $[x, u] \in D$; it is also sufficient for the optimality of $\left[x^{*}, u^{*}\right]$. 
Proof.- The necessity has been established with the previous sequence of propositions. Let us prove the sufficiency of the condition. Let $[x, u]$ be any feasible pair for $(P)$ and add (2.19) and (2.20) :

$\int_{0}^{T}\left\langle x^{* \prime}-x^{\prime}+A x^{*}-A x, p^{*}+r^{*}\right\rangle_{V^{\prime} \times V} d t+\left\langle w_{2}, u^{*}-u\right\rangle_{L^{2}(0, T ; U)}-\int_{0}^{T}\left\langle B u^{*}-B u, r^{*}\right\rangle_{V^{\prime} \times V} d t \leq 0$.

As $[x, u]$ is feasible we get

$$
\left\langle w_{2}, u^{*}-u\right\rangle_{L^{2}(0, T ; U)}+\int_{0}^{T}\left\langle x^{* \prime}-x^{\prime}+A x^{*}-A x, p^{*}\right\rangle_{V^{\prime} \times V} d t \leq 0 .
$$

Integrating by parts and taking in account the adjoint equation, we obtain :

$$
\left\langle w_{2}, u^{*}-u\right\rangle_{L^{2}(0, T ; U)}+\left\langle w_{1}, x^{*}-x\right\rangle_{L^{2}(0, T ; H)}+\left\langle w, x^{*}(T)-x(T)\right\rangle_{H} \leq 0 .
$$

The definition of the subdifferential achieves the proof.

Remark 2.4 To get a better insight of the relation (2.19), let us assume that $D=K \times U_{a d}$ (closed convex subsets in appropriate spaces). Let $r^{*}$ be in $\mathcal{X}$ (regularity). Then (2.19) can be written as following :

$$
\begin{aligned}
\int_{0}^{T} & \left\langle\partial_{x} L\left(x^{*}, u^{*}\right)-r^{* \prime}+A^{*} r^{*}, x^{*}-x\right\rangle_{V \times V^{\prime}} d t \\
& +\left\langle\partial l\left(x^{*}(T)\right)+r^{*}(T), x^{*}(T)-x(T)\right\rangle_{H} \leq 0,
\end{aligned}
$$

by partial integration and for any $x$ in $K$. If we consider the evolution system, which gives the adjoint equation of Barbu and Precupanu [3] :

$$
\begin{gathered}
\left.-\frac{d r^{*}}{d t}+A^{*}(t) r^{*}+\partial 1_{K}\left(x^{*}\right) \ni-\partial_{x} L\left(x^{*}, u^{*}\right) \text { a.e. in }\right] 0, T[, \\
r^{*}(T) \in-\partial l\left(x^{*}(T)\right),
\end{gathered}
$$

(where $1_{K}$ is the indicatrix function of $K$ ), then (2.21) is as a weak variant of (2.22)(2.23). In particular, when no state constraints are imposed, one may easily infer that $p^{*}=-r^{*}$. We see that condition $(\mathcal{H})$ yields the existence of a Lagrange multiplier, while $(\mathcal{S})$ ensures better regularity properties for it, (Barbu and Precupanu [3]).

Remark 2.5 The form of the optimality conditions may also be compared to the works of Bonnans and Casas [8, 9]. Basically, we decouple the influence of the state constraints from the adjoint equation and we put it as an independant inequality (2.19). The remaining simplified adjoint system (2.17)-(2.18) just performs the necessary integrations by parts in order to reexpress the gradient of the cost functional, and it is identical to the case without any state constraints (Lions [11]). This also avoids the delicate analysis of adjoint systems with measures as data, which is necessary when the classical approach is used (Casas [10]). 


\section{Some Applications}

Let $W \subset L^{2}\left(0, T ; V^{\prime}\right)$ continuously, densely be a Banach space. We replace $(\mathcal{H})$ by the weaker variant

$$
\begin{gathered}
\exists \mathcal{M} \subset D \text { bounded in } \mathcal{C}(0, T ; H) \times L^{2}(0, T ; U) \text { such that } \\
0 \in \operatorname{Int} T(\mathcal{M}) \text { in the } W \text { topology } .
\end{gathered}
$$

Since condition $(\mathcal{H})$ is used only in the proof of Proposition (2.4), then propositions 2.12.3 remain valid. We also ask the following pairing compatibility condition, which is automatically fulfilled in many examples :

$$
\langle v, w\rangle_{W \times W^{\prime}}=\int_{0}^{T}\langle v, w\rangle_{V \times V^{\prime}}
$$

when both terms have sense. We keep the notations of the proof of Proposition 2.4. Condition $\left(\mathcal{H}^{\prime}\right)$ yields that

$$
\rho\left\langle\xi, r_{\varepsilon}\right\rangle_{W \times W^{\prime}}<c
$$

that is $\left(r_{\varepsilon}\right)$ is bounded in the "larger" space $W^{\prime}$ instead of $L^{2}(0, T ; V)$. Let $r^{*}$ denote a weak * cluster point for this set.

Theorem 3.1 The pair $\left[x^{*}, u^{*}\right]$ is an optimal pair for $(P)$ if and only if

$$
\begin{gathered}
\left\langle w_{2}, u^{*}-u\right\rangle_{L^{2}(0, T ; U)}+\int_{0}^{T}\left\langle x^{* \prime}-x^{\prime}+A x^{*}-A x, p^{*}\right\rangle_{V^{\prime} \times V} d t \\
-\left\langle x^{\prime}+A x-B u-f, r^{*}\right\rangle_{W \times W^{\prime}} \leq 0,
\end{gathered}
$$

for any $[x, u]$ such that $T(x, u) \in W$.

Proof.- Necessity is a direct consequence of (2.7) and (3.2) since one may pass to the limit in all the terms if $T(x, u) \in W$.

For the sufficiency we notice that any feasible pair for $(P)$ satisfies $T(x, u)=0 \in W$. Then (3.3) may be used and only the first two terms will remain. The proof is finished as in Theorem 2.1.

Remark 3.1 In applications, $T(x, u) \in W$ may be valid for any pair $[x, u] \in D$ or this may be equivalent with a regularity condition which is possible to include in the definition of the state and control spaces. See [9] for the details of this technique in a different setting.

\subsection{A First Example : Empty Interior Constraints.}

We analyse in some detail the following example of optimal control problem governed by a parabolic partial differential equation :

$$
\operatorname{Min}\left\{\frac{1}{2} \int_{Q}\left(y-z_{d}\right)^{2} d x d t+\frac{N}{2} \int_{Q} u^{2} d x d t\right\}
$$


subject to :

$$
\begin{aligned}
& \left.\frac{\partial y}{\partial t}-\Delta y=f+u \quad \text { in } Q=\Omega \times\right] 0, T[, \\
& y(x, t)=0 \quad \text { on } \Sigma=\partial \Omega \times] 0, T[, \\
& y(x, 0)=y_{o}(x) \text { in } \Omega \text {, }
\end{aligned}
$$

and the constraints

$$
\begin{array}{ll}
e(x, t) \leq y(x, t) \leq g(x, t) & \text { a.e. in } Q \\
a(x, t) \leq u(x, t) \leq b(x, t) & \text { a.e. in } Q
\end{array}
$$

Here $\Omega$ is a smooth, open and bounded domain of $\mathbb{R}^{n}, z_{d} \in \operatorname{Leuxq}, N \geq 0, y_{o} \in$ Leux, $f, a, b$ are in $L^{\infty}(Q)$ and $e, g$ in $\mathcal{C}(\bar{Q})$. We denote

$$
\begin{gathered}
K=\left\{y \in L^{2}\left(0, T ; H_{o}^{1}(\Omega)\right) \cap W^{1,2}\left(0, T ; H^{-1}(\Omega)\right) \mid e \leq y \leq g, y(., 0)=y_{o}\right\}, \\
U_{a d}=\{u \in \operatorname{Leuxq} \mid a \leq u \leq b \text { a.e. in } Q\} \\
D=K \times U_{a d},
\end{gathered}
$$

which are closed convex sets. One has to assume the compatibility condition

$$
e \leq y^{o} \leq g \quad \text { in } \Omega,
$$

and some admissibility hypothesis. We ask

$$
\exists \alpha>0, \exists \tilde{u} \in U_{a d} \quad \text { such that } \quad e \leq Y(\tilde{u}-\alpha) \leq Y(\tilde{u}+\alpha) \leq g \quad \text { in } Q,
$$

where $Y$ is the solution operator $u \mapsto y$ defined by (3.4)-(3.6). By comparison, $(\mathcal{E})$ implies that the pair $[\tilde{u}, Y(\tilde{u})]$ is feasible for $(P P)$. However, this is not an interiority assumption since $e$ may be equal to $g$ in some points. For instance, we may allow $e(x, t)=g(x, t)=0$ on the border of the domain. Moreover, the mappings $\tilde{u}+\alpha, \tilde{u}-\alpha$ need not belong to $U_{a d}$, which may also have a void interior, i.e $a=b$ on some subset.

Remark 3.2 A stronger variant of $(\mathcal{E})$ is : there exist two controls $\tilde{u}, \hat{u}$ feasible for $(P P)$, which can be "strictly separated". It means that $(\mathcal{E})$ is stronger than the standard admissibility assumption, but weaker than the hypothesis of existence of two feasible pairs (with this separation property). As $(\mathcal{E})$, in turn, yields $\left(\mathcal{H}^{\prime}\right)$, this gives a hint on the generality of the hypothesis $\left(\mathcal{H}^{\prime}\right)$. Moreover $\left(\mathcal{H}^{\prime}\right)$ requires that the problem $(P)$ is non trivial, that is the set of admissible pairs is "rich".

In order to apply the abstract theory, we take the spaces $V=H_{o}^{1}(\Omega), H=U=$ Leux, the operators $A(t): V \rightarrow V^{\prime}, A(t) z=-\Delta z, B: H \rightarrow V^{\prime}, B=i$, the canonical injection, and the mappings $l=0$ and

$$
L(y, u)=\frac{1}{2} \int_{Q}\left(y-z_{d}\right)^{2} d x d t+\frac{N}{2} \int_{Q} u^{2} d x d t .
$$

The hypothesis $\left(\mathcal{H}^{\prime}\right)$ is a clear consequence of $(\mathcal{E})$ with $W=L^{\infty}(Q)$, by fixing

$$
y_{\xi}=Y\left(u_{\xi}\right)=Y(\tilde{u}+\alpha \xi), \quad \xi \in W,\|\xi\|_{W}=1 .
$$


Again a comparison argument shows that $\left[y_{\xi}, u_{\xi}\right] \in D$ for any $\xi$ as above and we can choose in $\left(\mathcal{H}^{\prime}\right)$ the bounded set

$$
\mathcal{M}=\operatorname{conv}\left\{\left[y_{\xi}, u_{\xi}\right] \mid \xi \in W,\|\xi\|_{W}=1\right\} .
$$

Then relation (3.3) may be rewritten as :

$$
\begin{aligned}
\int_{Q} N u^{*}\left(u^{*}-u\right) d x d t+\int_{Q}\left(y^{* \prime}-y^{\prime}-\Delta\left(y^{*}-y\right)\right) p^{*} d x d t & \\
-\left\langle y^{\prime}-\Delta y-u-f, r^{*}\right\rangle_{W \times W^{\prime}} & \leq 0,
\end{aligned}
$$

for any $y$ in $K, u$ in $U_{a d}$ such that $T(y, u) \in W=L^{\infty}(Q)$.

Notice also that, since $f \in W, U_{a d} \subset W$, the last condition $\left(T(y, u) \in W=L^{\infty}(Q)\right)$ is equivalent to a regularity condition on $y: y^{\prime}-\Delta y \in L^{\infty}(Q)$, which is satisfied by $y^{*}$. Here $\left[y^{*}, u^{*}\right]$ is the optimal pair of $(P P)$ and $p^{*}$ satisfies

$$
\begin{aligned}
-p^{* \prime}-\Delta p^{*} & =y^{*}-z_{d} & & \text { in } Q \\
p^{*} & =0 & & \text { on } \Sigma \\
p^{*}(T) & =0 & & \text { in } \Omega .
\end{aligned}
$$

Choosing in turn $u=u^{*}$ and $y=y^{*}$ in (3.9), we get

$$
\begin{gathered}
\forall y \in K \text { such that } y^{\prime}-\Delta y \in L^{\infty}(Q) \quad\left\langle y^{* \prime}-y^{\prime}-\Delta\left(y^{*}-y\right), p^{*}+r^{*}\right\rangle_{W \times W^{\prime}} \leq 0, \\
\forall u \in U_{a d} \quad\left\langle N u^{*}-r^{*}, u^{*}-u\right\rangle_{W \times W^{\prime}} \leq 0
\end{gathered}
$$

The relations (3.10) and (3.11) represent the decoupled form of the optimality condition (3.9) and may be compared to (2.19) and (2.20).

Now, we are going to use these above relations to get some more precise information about the optimal pair. Let us define the sets

$$
\begin{gathered}
Q_{e}=\left\{(x, t) \in \bar{Q} \mid y^{*}(x, t)=e(x, t)\right\}, Q_{g}=\left\{(x, t) \in \bar{Q} \mid y^{*}(x, t)=g(x, t)\right\}, \\
Q^{o}=Q-\left(Q_{e} \cup Q_{g}\right) .
\end{gathered}
$$

Thanks to (3.4)-(3.6) and the Sobolev embedding theorem, we infer that $y^{*} \in \mathcal{C}(\bar{Q})$ if $y_{o}$ is regular. Then $Q_{e}$ and $Q_{g}$ are closed sets and $Q^{o}$ is an open subset of $Q$. Let $d \in \mathcal{D}(Q)$ be a test function with compact support supp $d \subset Q^{o}$. By the continuity of $y^{*}, e, g$ and the compactness of supp $d$, one can find $\rho>0$ such that $y^{*}+\rho d$ and $y^{*}-\rho d$ remain in $K$. Obviously, they are also regular and we can use them in (3.10) as test functions to infer :

$$
\left\langle p^{*}+r^{*}, \frac{\partial d}{\partial t}-\Delta d\right\rangle_{W \times W^{\prime}}=0
$$

for any $d \in \mathcal{D}(Q)$ with compact support in $Q^{o}$. Taking in account this relation and the equation satisfied by $p^{*}$, we see that there exists a distribution $j \in \mathcal{D}^{\prime}(Q)$ with support included in $Q-Q^{o}$, such that

$$
\frac{\partial r^{*}}{\partial t}-\Delta r^{*}+j=y^{*}-z_{d} \quad \text { in } \mathcal{D}^{\prime}(Q)
$$


The previous equation is another familiar form of the adjoint system for state constrained control problems. In particuler, it shows that $r^{*} \in W_{l o c}^{2,1, p}\left(Q^{o}\right)$ for $p>1$ if $z_{d}$ belongs to $L^{p}(Q)$. Then $r^{*} \in \mathcal{C}\left(Q^{o}\right)$ by the Sobolev theorem if $p$ is big enough.

We are now prepared to give a result on the structure of the optimal pair of $(P P)$, which may be termed as a generalized bang-bang result, Tröltzsch [16]. We suppose from now that $\mathrm{N}=0$.

Corollary 3.1 We have :

$Q^{o} \subseteq\left\{(x, t) \mid y^{*}(x, t)=z_{d}(x, t)\right\} \cup\left\{(x, t) \mid u^{*}(x, t)=a(x, t)\right\} \cup\left\{(x, t) \mid u^{*}(x, t)=b(x, t)\right\}$.

Proof.- Choose $u=u^{*}$ in $Q-Q^{o}$ so that (3.11) yields

$$
\forall u \in U_{a d} \quad \int_{Q^{o}} r^{*}\left(u^{*}-u\right) d x d t \geq 0
$$

Since $r^{*} \in \mathcal{C}\left(Q^{o}\right)$, obviously

$Q^{o}=\left\{(x, t) \in Q^{o} \mid r^{*}(x, t)>0\right\} \cup\left\{(x, t) \in Q^{o} \mid r^{*}(x, t)<0\right\} \cup\left\{(x, t) \in Q^{o} \mid r^{*}(x, t)=0\right\}$,

and (3.13) shows that $u^{*}=b$ on the first set and $u^{*}=a$ on the second set. If the last set has positive measure, then (3.12) and the maximal regularity of $r^{*}$ on $Q^{o}$ gives that $y^{*}=z_{d}$ on this subset.

Remark 3.3 Taking into account the definition of $Q^{o}$, we see that at least one from $y^{*}$ and $u^{*}$ equals the extremal values $e, g, a, b$ or $z_{d}$ in any point of $Q$. A similar analysis may be pursued when $N>0$, but the structure of the optimal pair will be more complicated.

\subsection{A Second Example :"Bottleneck" Problems.}

We examine "bottleneck" type problems which were introduced by Bellman [4] in connection with some models for industrial production processes. They were discussed by a different approach in the work of Miricà [13].

We assume that the state equation and the cost functional are the same as in the previous example, with $f \in L^{\infty}(Q), y_{o} \in W_{o}^{1, \infty}(\Omega) \cap W^{2, \infty}(\Omega), y_{o} \geq 0$ a.e. in $\Omega$, but the constraint has the form

$$
|y| \leq u \quad \text { a.e. in } Q \text {. }
$$

This is equivalent with : $-u \leq y \leq u$, so that the set $D$ defined by (3.14) is convex.

Remark 3.4 If $f \geq 0$, the maximum principle gives $y \geq 0$ (with (3.4)) and the constraint (3.14) is equivalent to

$$
0 \leq y \leq u \quad \text { a.e. in } Q
$$

which is the original form considered by Bellman [4]. We also underline that the boundary condition (3.5) shows that the feasible pairs are not in the interior of $D$ even in the $L^{\infty}(Q)$-topology, that is Slater-type conditions cannot be valid in (3.14'). 
Take now $\tilde{u}=\alpha e^{t}, \alpha>0$ and $\tilde{y}$ the solution of (3.4)-(3.6) associated to $\tilde{u}$. If $\alpha$ is great enough, then $f+\tilde{u} \geq 0$ a.e. in $Q$ and $\tilde{y} \geq 0$ a.e in $Q$ by the maximum principle. Let $w=\alpha e^{t}-\alpha \geq 0$ a.e in $Q$. We notice that $w$ satisfies

$$
\begin{aligned}
& \frac{\partial w}{\partial t}-\Delta w=\alpha e^{t} \quad \text { in } Q \\
& w \quad=\left(\alpha e^{t}-\alpha\right)_{\mid \Sigma} \geq 0 \text { on } \Sigma \\
& w(0)=0 \quad \text { in } \Omega \text {. }
\end{aligned}
$$

Let us denote $y^{v}$ the solution of (3.4)-(3.6) associated to $v$ (so that $y^{o}$ is associated to $v=0)$. By comparison, it yields that $w \geq \tilde{y}-y^{o}$, that is $\tilde{u}-\alpha+y^{o} \geq \tilde{y} \geq 0$. There is some constant $m$ such that $-m \leq y^{o} \leq m$ a.e in $Q$. Then, if $\alpha>2 m$ is large enough, we have

$$
0 \leq \tilde{y} \leq \tilde{u}-m \quad \text { a.e in } Q .
$$

The pair $[\tilde{y}, \tilde{u}]$ is feasible and (3.16) shows that hypothesis $\left(\mathcal{H}^{\prime}\right)$ is satisfied. Indeed we take

$$
\mathcal{M}=\left\{\left[y^{v}, \tilde{u}\right] \mid v \in B_{L^{p}(Q)}(\tilde{u}, \lambda)\right\}, p>\frac{n+2}{2},
$$

where $B_{L^{p}(Q)}(\tilde{u}, \lambda)$ is the $L^{p}$-ball centered in $\tilde{u}$ with radius $\lambda$, and $\lambda>0$ is small. By the continuity with respect of the right-hand side and the Sobolev embedding theorem, we can choose $\lambda$ such that

$$
\left\|\tilde{y}-y_{v}\right\|_{\mathcal{C}(\bar{Q})} \leq m
$$

that is $\mathcal{M} \subset D$. Moreover $T(\mathcal{M})=B_{L^{p}(Q)}(0, \lambda)$ and $\left(\mathcal{H}^{\prime}\right)$ is fulfilled in $L^{p}(Q)$. If $n=1$ we may take $p=2$ and even condition $(\mathcal{H})$ is fulfilled.

Remark 3.5 We also notice that for the "linear" constraint (3.14') a simpler argument may be used. For instance, we may define the new control function

$$
\varphi=y-u,
$$

and D may be reexpressed in the "decoupled" form

$$
y \geq 0, \varphi \leq 0, \text { a.e in } Q \text {. }
$$

The same substitution may be performed in (3.4)-(3.6) and in the cost functional, so that the penalization method from section 2. may be used directly.

Finally, we prove that a generalized bang-bang result remains valid in this case as well, under some regularity assumptions.

Corollary 3.2 Let $N$ be equal to 0 and assume that $y^{*}$, $u^{*}$ exist and are continuous functions on $\bar{Q}$. Then

$$
Q=\left\{(x, t) \in Q|| y^{*}(x, t) \mid=u^{*}(x, t)\right\} \cup\left\{(x, t) \in Q \mid y^{*}(x, t)=z_{d}(x, t)\right\} .
$$


(The two sets above need not be disjoint. The first one corresponds to the case where the constraint is active.)

Proof.- As hypothesis $\left(\mathcal{H}^{\prime}\right)$ is fulfilled, theorem (3.1) gives the existence of $r^{*} \in L^{q}(Q)$ where $\frac{1}{p}+\frac{1}{q}=1$ and :

$$
\int_{Q}\left(y^{* \prime}-y^{\prime}-\Delta\left(y^{*}-y\right)\right) p^{*} d x d t-\int_{Q}\left(y^{\prime}-\Delta y-u-f\right) r^{*} d x d t \leq 0
$$

for any $[y, u] \in D$ such that $T(y, u) \in L^{p}(Q)$.

Here we used that $N=0$ and $p^{*}$ is given by

$$
\begin{aligned}
& -p^{* \prime}-\Delta p^{*}=y^{*}-z_{d} \text { in } Q \\
& p^{*} \quad=\quad 0 \text { on } \Sigma \\
& p^{*}(T)=0 \quad \text { in } \Omega \text {. }
\end{aligned}
$$

Let $Q^{*} \subset Q$ be the open set defined as following

$$
Q^{*}=\left\{(x, t) \in Q|| y^{*}(x, t) \mid<u^{*}(x, t)\right\},
$$

where the constraint is inactive. First we take test pairs of the type

$$
[y, u]=\left[y^{*} \pm \lambda_{d} d, u^{*}\right] \in D,
$$

where $d \in \mathcal{D}(Q)$, supp $d \subset Q^{*}$ and $\lambda_{d}>0$ is a small constant given by the Weierstrass theorem applied to the continuous functions $\left|y^{*}\right|, u^{*}$ on the compact set supp $d$, such that

$$
\left|y^{*}(x, t) \pm \lambda_{d} d(x, t)\right| \leq\left|y^{*}(x, t)\right|+\lambda_{d}|d(x, t)| \leq u^{*}(x, t) .
$$

Thanks to (3.18), we obtain after a short calculation, that

$$
\int_{Q}\left(d^{\prime}-\Delta d\right)\left(p^{*}+r^{*}\right) d x d t=0
$$

for every $d \in \mathcal{D}(Q)$ with compact support in $Q^{*}$. Then, we may find a distribution $j \in \mathcal{D}^{\prime}(Q)$ with support in $Q-Q^{*}$ (active constraints set) such that :

$$
\frac{\partial r^{*}}{\partial t}+\Delta r^{*}+j=y^{*}-z_{d} \quad \text { in } \mathcal{D}^{\prime}(Q)
$$

This follows from (3.19) and (3.20), and it implies a local regularity property for the Lagrange multiplier $r^{*}: r^{*} \in W_{l o c}^{2,1,2}\left(Q^{*}\right)$ since $z_{d} \in L^{2}(Q)$.

Now let us take the test pairs

$$
[y, u]=\left[y^{*}, u^{*} \pm \lambda_{d} d\right] \in D,
$$

where $d, \lambda_{d}$ are as above. Again, by (3.18) we get

$$
\int_{Q} d r^{*} d x d t=0
$$


Multiplying (3.21) by $d$, we infer

$$
\int_{Q} d\left(y^{*}-z_{d}\right) d x d t=\left\langle r^{* \prime}+\Delta r^{*}, d\right\rangle_{\mathcal{D}^{\prime}(Q) \times \mathcal{D}(Q)}=-\int_{Q} r^{*}\left(d^{\prime}-\Delta d\right) d x d t=0,
$$

for any $d \in \mathcal{D}(Q)$ with support in $Q^{*}$. This proves that $y^{*}=z_{d}$ in $Q^{*}$ and the proof is over.

\section{Acknowledgement}

Part of this work has been done during the stay of the second author at Technische Universität München under the support of the Alexander von Humboldt Foundation. The second author thanks the Foundation for the support.

\section{References}

[1] V. Barbu, Optimal Control of Variational Inequalities, Research Notes in Mathematics 100, Pitman, London (1984).

[2] V.Barbu-N.Pavel, Optimal control problems with two point boundary condition, Journal of Optimization Theory and Applications, Vol.77, pp.51-78 (1993).

[3] V. Barbu - Th. Precupanu, Convexity and optimization in Banach spaces, Sijthoff and Noordhoff, Leyden (1978).

[4] R. Bellman, Dynamic Programming, Princeton University Press (1957).

[5] M. Bergounioux, Contrôle Optimal de Problèmes Elliptiques avec Contraintes sur l'Etat, Comptes Rendus de l'Académie des Sciences, t 310, série I , pp. 391-396 , (1990).

[6] _ A Penalization Method for Optimal Control of Elliptic Problems with State Constraints, SIAM Journal on Control and Optimization - Vol $30, \mathrm{n}^{\mathrm{O}} 2$, pp. 305-323,(1992).

[7] M. Bergounioux - T. Männikkö - D. Tiba, On Nonqualified Parabolic Control Problems, Preprint nº 148, University of Jyväskylä, Finland (July 1992).

[8] J.F. Bonnans - E. Casas, Optimal control of semilinear multistate systems with state constraints, SIAM Journal on Control and Optimization - Vol $27, \mathrm{n}^{\mathrm{o}} 2$, pp.446$455,(1989)$.

[9] problems, Numerical Functional Analysis and Optimization 7 (4), pp. 333-348 (1984$85)$.

[10] E. Casas, Boundary control of semilinear elliptic equations with pointwise state constraints, SIAM Journal on Control and Optimization, vol.31, $\mathrm{n}^{\mathrm{o}} 4$, pp. 993-1006 (1993).

[11] J.L. Lions, Contrôle optimal des systèmes gouvernés par des équations aux dérivées partielles, Dunod, Paris (1968). 
[12] _ Contrôle des systèmes distribués singuliers, Gauthier-Villars, Paris (1983).

[13] S. Miricà, Optimal feedback control for a class of bottleneck problems, Journal Mathematical Analysis and Applications, 112, $\mathrm{n}^{\mathrm{o}}$ 1, pp. 221-235 (1985).

[14] D. Tiba, Optimal control of nonsmooth distributed parameter systems, Lecture Notes $\mathrm{n}^{\mathrm{o}}$ 1459, Springer-Verlag, Berlin (1990).

[15] V. Tikhomirov, Fundamental principles of the theory of extremal problems, J.Wiley, Chichester, (1986).

[16] F. Tröltzsch, Optimality conditions for parabolic control problems and applications, Teubner Texte, Leipzig, (1984).

[17] _ A modification of the Zowe and Kurcyusz regularity condition with application to the optimal control of Noether operator equations with constraints on the control and the state, Math. Operationforsch. Statist., Ser. Optimization, vol.14, $\mathrm{n}^{\mathrm{O}} 2$, pp.245-253 (1983).

[18] J. Zowe - S. Kurcyusz, Regularity and stability for the mathematical programming problem in Banach spaces, Applied mathematics and Optimization, 5, pp.49-62 (1979). 